Arq. Bras. Med. Vet. Zootec., v.71, n.4, p.1179-1186, 2019

\title{
Morfometria dos meniscos lateral e medial na articulação femorotibial de equinos
}

[Morphometry of the lateral and medial meniscus in the equine femorotibial joint]

${ }^{1}$ Residência Multiprofissional - Hospital Veterinário da Universidade Federal do Paraná - Curitiba, PR

${ }^{2}$ Aluno de graduação - Universidade Federal do Paraná - Curitiba, PR

${ }^{3}$ Universidade Federal do Paraná - Curitiba, PR

\begin{abstract}
RESUMO
As lesões dos meniscos são uma das principais causas de dor e claudicação em equinos. O objetivo do presente estudo foi avaliar a morfometria dos meniscos da articulação femorotibial de equinos. Foram utilizados 48 meniscos de 12 animais de ambos os sexos, com idade entre cinco e 15 anos e com peso entre $400 \mathrm{~kg}$ e $500 \mathrm{~kg}$. A medida da extensão periférica compreendeu desde a parte mais cranial à mais caudal e denominou-se circunferência externa (CE). A margem interna, com o mesmo tratamento, foi chamada de circunferência interna (CI). Os meniscos foram divididos em terços craniais, médios e caudais. A espessura foi obtida nos pontos médios de cada terço. Foi calculada a área dos meniscos em contato com os côndilos femorais. O menisco medial apresentou maior CE com média de 126,38mm, enquanto o menisco lateral apresentou média de $115,32 \mathrm{~mm}$. O menisco lateral mostrou maior espessura nos terços médio e caudal, com valores médios de $16,00 \mathrm{~mm}$ e $19,85 \mathrm{~mm}$, respectivamente, contra $13,75 \mathrm{~mm}$ e $14,99 \mathrm{~mm}$ dos meniscos mediais. Os resultados deste estudo mostraram relação importante entre os dados morfométricos e os achados clínicos na tentativa de explicar a maior incidência de lesões envolvendo o menisco medial.
\end{abstract}

Palavras-chave: cavalo, joelho, menisco, claudicação

\begin{abstract}
Meniscal lesions are one of the main causes of pain and lameness in horses. The objective of this study was to evaluate the morphometry of the meniscus of the femorotibial joint of horses. 48 meniscus were used from 12 animals, aged between five and 15 years and weighing between 400kg and 500kg. The measurement of the peripheral extension went from the most cranial part to the most caudal, denominated external circumference $(C E)$. The internal margin, with the same treatment, was called internal circumference (IC). The meniscus was divided into cranial, middle and caudal thirds. The thickness was obtained at the midpoints of each third. The area of the meniscus in contact with the femoral condyles was also calculated. The medial meniscus presented a higher CE with a mean of $126.38 \mathrm{~mm}$, while the lateral meniscus presented a mean of $115.32 \mathrm{~mm}$. The lateral meniscus showed greater thickness in the middle and caudal thirds, with mean values of $16.00 \mathrm{~mm}$ and $19.85 \mathrm{~mm}$ respectively, against $13.75 \mathrm{~mm}$ and $14.99 \mathrm{~mm}$ of the medial meniscus. The results of this study showed an important relationship between the morphometric data and the clinical findings in an attempt to explain the higher incidence of lesions involving the medial meniscus.
\end{abstract}

Keywords: horse, knee, meniscus, lameness

\section{INTRODUÇÃO}

Lesões às fibrocartilagens dos meniscos da articulação femorotibial são comuns em equinos, caninos e humanos. Independentemente da

Recebido em 28 de dezembro de 2017

Aceito em 10 de dezembro de 2018

E-mail: altinabruna@gmail.com espécie, lesões em estruturas avasculares, como nos meniscos, são de difícil controle por serem incapazes de sofrer uma reparação espontânea, necessitando, de acordo com o tipo de lesão, de uma abordagem cirúrgica por meio de meniscectomia parcial (Arnoczky e Warren, 1983). 
Lesões de menisco em cavalos foram descritas inicialmente em 1978 por Valdez e Adams, e atualmente a literatura é constituída predominantemente por estudos retrospectivos (Walmsley, 1995). Em um estudo, notou-se que, em 110 casos de lesões de menisco diagnosticadas, $79 \%$ envolviam o corno cranial do menisco medial, ao contrário do cão ou do homem nos quais essas lesões são geralmente associadas ao ligamento cruzado e/ou ligamentos colaterais (Almeida et al., 2004; Walmsley, 2005; Murlimanju et al., 2010). Danos no menisco medial têm sido associados também a lesões císticas subcondrais no côndilo femoral medial (Hendrix et al., 2010).

Estudos anatômicos e morfométricos têm sido realizados em ligamentos com inserção nos meniscos, com o objetivo de avaliar suas funções na estabilização da articulação femorotibial, o que poderia prevenir lesões por translocação dos meniscos. Entre os ligamentos citados, os autores fazem referência ao ligamento meniscofemoral caudal e meniscotibial cranial (Gupte et al., 2007; Fowlie et al., 2011; Fowlie et al., 2012; Oliveira et al., 2013). Para Fowlie et al. (2012), o ligamento meniscotibial cranial pode ter uma relação às lesões que ocorrem no corno cranial do menisco medial de equinos.

Neste estudo objetivou-se avaliar os aspectos morfométricos relacionados aos meniscos lateral e medial da articulação femorotibial de equinos para elucidar possíveis causas anatômicas para a maior incidência de lesões envolvendo o menisco medial nessa espécie.

\section{MATERIAL E MÉTODOS}

Foram avaliados meniscos de 24 equinos da raça Puro Sangue Inglês, de ambos os sexos, com idade entre cinco e 15 anos, e peso entre 400 e $500 \mathrm{~kg}$, totalizando 48 meniscos mediais (MM) e 48 meniscos laterais (ML). Os meniscos estudados foram retirados de animais submetidos à eutanásia no Hospital Veterinário da Universidade Federal do Paraná, sendo excluídos equinos que apresentavam histórico de claudicação e doença articular em qualquer região do sistema locomotor. As peças foram analisadas e mensuradas no Departamento de Anatomia do Setor de Ciências Biológicas da Universidade Federal do Paraná e da disciplina de Anatomia Patológica do Departamento de Medicina Veterinária do Setor de Ciências Agrárias da Universidade Federal do Paraná.

As articulações foram fixadas em formaldeído a $10 \%$ e, após sete dias, lavadas em água corrente e dissecadas. Para exposição dos meniscos, a cápsula articular foi seccionada e retirada, possibilitando a exposição dos ligamentos transversos, do corno cranial e do corno caudal dos meniscos medial e lateral. Após disseção, foram realizadas mensurações de comprimento, largura e espessura dos ligamentos supracitados. $\mathrm{Na}$ sequência, os ligamentos intra-articulares foram seccionados para o afastamento dos côndilos femorais, para a exposição e remoção dos meniscos. Todas as dissecções foram padronizadas. A extensão periférica dos meniscos foi obtida por meio de fio de cobre posicionado ao longo da margem externa de cada menisco e fixado por pinos metálicos. A medida da extensão compreendeu da parte mais cranial, ápice do corno cranial, à parte mais caudal, ápice do corno caudal, e foi denominada “circunferência externa" (CE). Do mesmo modo, a margem interna recebeu o mesmo tratamento e foi chamada de "circunferência interna" (CI) (Fig. 1).

Os meniscos lateral e medial foram divididos em três segmentos iguais: terço cranial (TCr), envolvendo os cornos craniais, terço médio (TM) e terço caudal (TCa) com os cornos caudais (Fig. 1). Os pontos médios de cada terço foram submetidos a mensurações para a obtenção da largura e da espessura. Além dessas, foram também calculadas as áreas dos meniscos em contato com os côndilos femorais e as áreas dos côndilos das tíbias em contato com os meniscos. Para isso, os meniscos e as tíbias foram fotografados dorsalmente, e as imagens foram analisadas no programa de computador ImageJ 1.4.3.67 Launcher Simmetry Software. 
(a)

(b)
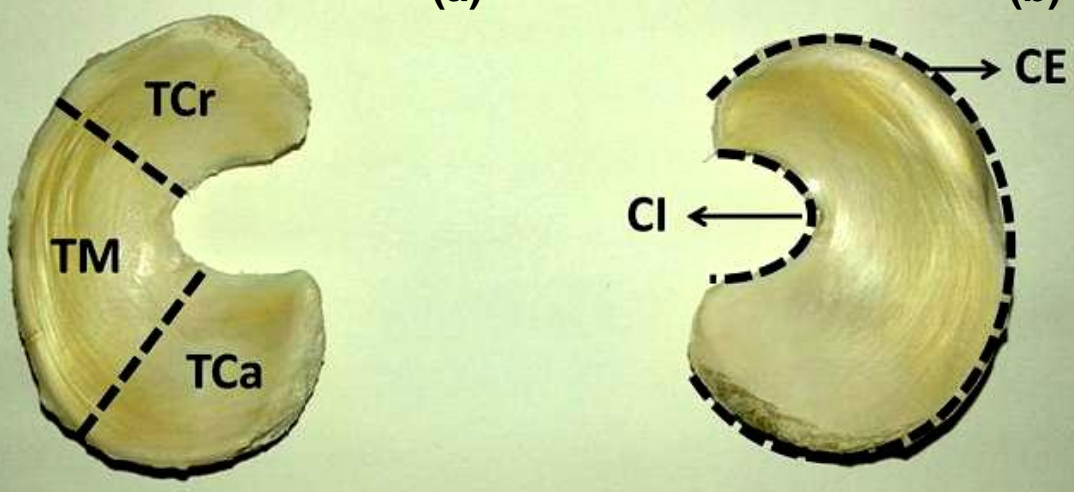

Figura 1. Método utilizado para divisão dos segmentos dos meniscos lateral e medial de equino: (a) terço cranial (TCr), terço médio (TM) e terço caudal (TCa); (b) mensuração da circunferência externa (CE) e da circunferência interna $(\mathrm{CI})$.

As medições foram obtidas por meio de paquímetro digital com precisão centesimal (modelo Starret - Vernier Caliper 125B, Stainless Hardned 93/116 1385 - Starret Indústria e Comércio Ltda., Itu, SP, Brasil). Os dados obtidos foram submetidos à análise estatística pelo programa de computador StatView 5.0.1 (SAS Institute Inc., NC, USA). O teste Kolmogorov-Smirnov foi utilizado para verificar a distribuição normal dos dados. Os dados paramétricos foram submetidos ao teste $\mathrm{T}$ de Student não pareado, com grau de significância de $5 \%$.

\section{RESULTADOS}

Com base nas medições realizadas nos 48 meniscos mediais (MM) e nos 48 meniscos laterais (ML), foram analisados os dados $\mathrm{e}$ calculados os valores médios de cada parâmetro e seus respectivos desvios-padrão (Tab. 1).

O menisco medial apresentou maior $\mathrm{CE}$, com média de $126,38 \mathrm{~mm}$, enquanto o menisco lateral apresentou média de $115,32 \mathrm{~mm}$, com diferença estatística significante $(\mathrm{P}<0,05)$. Não houve diferença na CI entre os meniscos (Fig. 2).

Tabela 1. Valores médios obtidos na mensuração dos meniscos lateral e medial (CE - circunferência externa; CI - circunferência interna; ETCr - espessura do terço cranial; ETM - espessura do terço médio; ETCa - espessura do terço caudal; LTCr - largura do terço cranial; LTM - largura do terço médio; LTCa - largura do terço caudal; AT - área total; ATCr - área do terço cranial)

\begin{tabular}{lcc} 
& Menisco lateral & Menisco medial \\
\hline CE $*$ & $115,820 \pm 8,705$ & $126,484 \pm 8,423$ \\
CI & $36,674 \pm 2,401$ & $41,605 \pm 7,187$ \\
ETCr & $14,733 \pm 1,388$ & $14,730 \pm 1,549$ \\
ETM $*$ & $15,707 \pm 1,698$ & $13,415 \pm 1,331$ \\
ETCa $*$ & $19,297 \pm 3,084$ & $15,351 \pm 1,641$ \\
LTCr & $19,331 \pm 2,331$ & $19,185 \pm 1,057$ \\
LTM $*$ & $21,067 \pm 2,271$ & $19,224 \pm 2,333$ \\
LTCa & $21,378 \pm 2,785$ & $21,464 \pm 2,342$ \\
AT & $16,133 \pm 2,567$ & $16,784 \pm 2,345$ \\
ATCr $*$ & $2,463 \pm 0,480$ & $3,140 \pm 0,418$ \\
\hline
\end{tabular}

*Apresentaram diferença estatisticamente significante. 


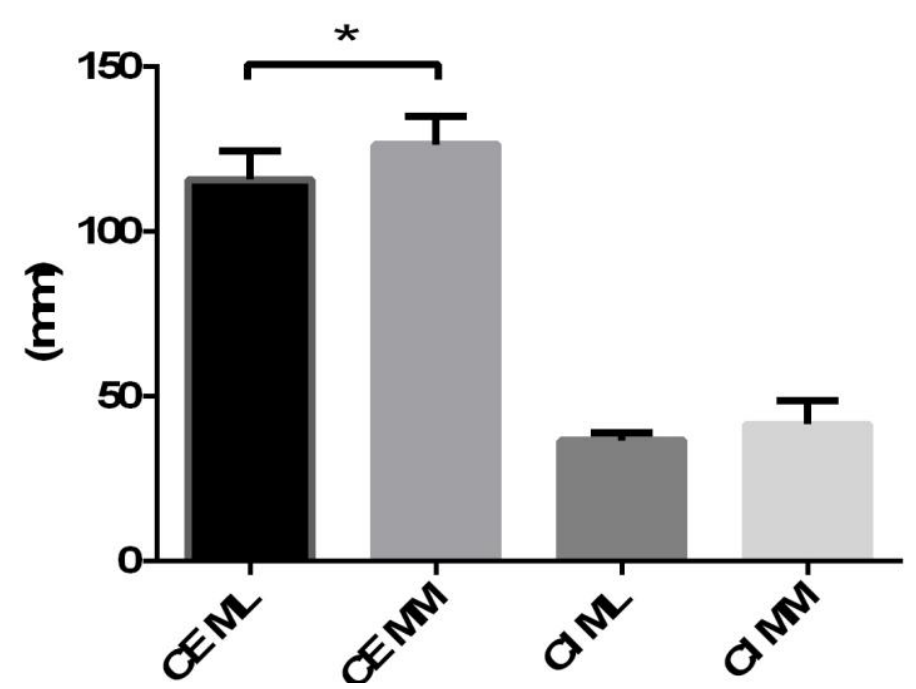

Figura 2. Valores médios e desvio-padrão das medidas de meniscos lateral e medial de equino, em que foram avaliadas: circunferência externa de menisco lateral (CE ML), circunferência externa de menisco medial (CE MM), circunferência interna de menisco lateral (CI ML) e circunferência interna de menisco medial (CI MM).

O menisco lateral mostrou maior espessura nos terços médio e caudal (Fig. 3), com valores médios de 16,00mm e 19,85mm, respectivamente, contra $13,75 \mathrm{~mm}$ e $14,99 \mathrm{~mm}$ dos meniscos mediais, estatisticamente significante, com $\mathrm{P}<0,05$. O terço cranial não mostrou diferença em relação à espessura.
$\mathrm{O}$ menisco lateral apresentou maior largura no ponto médio do terço médio (Fig. 4), com valor médio de $21,06 \mathrm{~mm}$ contra $19,22 \mathrm{~mm}$ do menisco medial, estatisticamente significante, com $\mathrm{P}<0,05$. Os terços cranial e caudal não apresentaram diferenças em relação à largura.

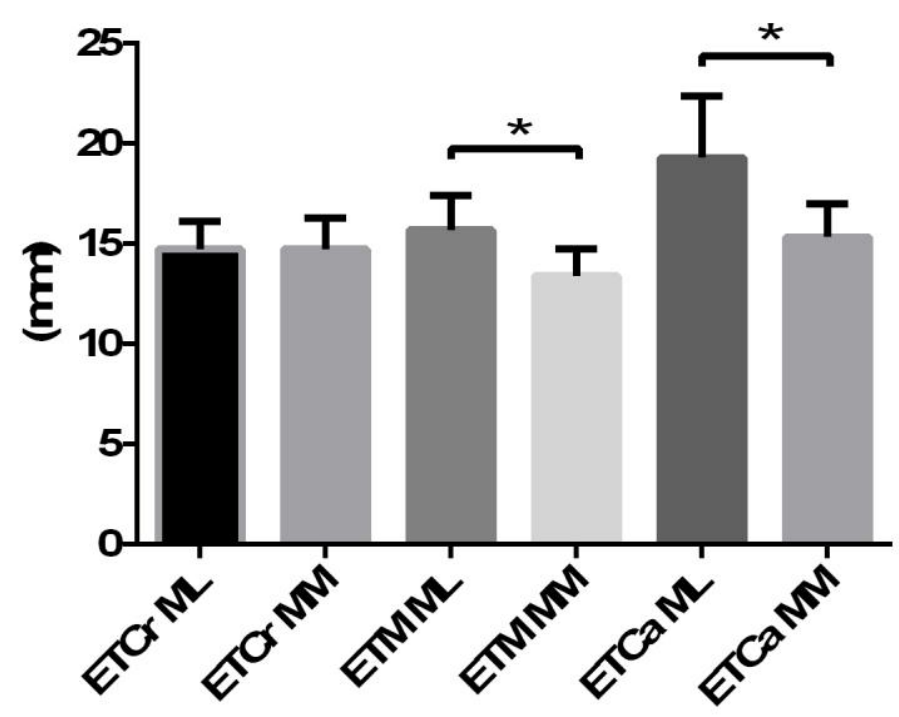

Figura 3. Médias e desvio-padrão das medidas de meniscos lateral e medial de equino, em que foram avaliadas: espessura do terço cranial do menisco lateral (ETCr ML), espessura do terço cranial do menisco medial (ETCr MM), espessura do terço médio do menisco lateral (ETM ML), espessura do terço médio do menisco medial (ETM MM), espessura do terço caudal do menisco lateral (ETCa ML), espessura do terço caudal do menisco medial (ETCa MM). 


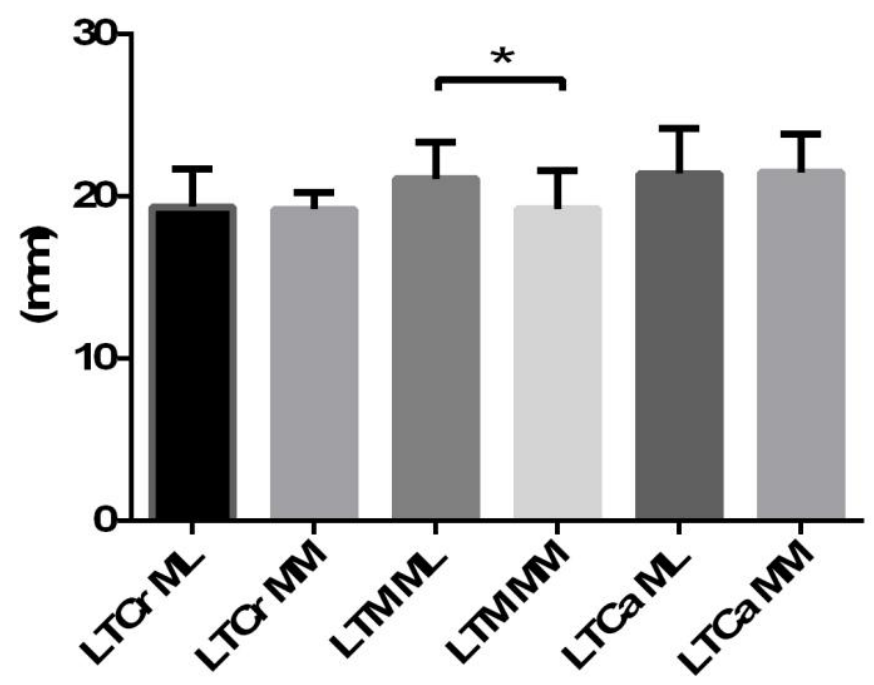

Figura 4. Valores médios e desvio-padrão das medidas de meniscos lateral e medial de equino, em que foram avaliadas: largura do terço cranial do menisco lateral (LTCr ML), largura do terço cranial do menisco medial (LTCr MM), largura do terço médio do menisco lateral (LTM ML), largura do terço médio do menisco medial (LTM MM), largura do terço caudal do menisco lateral (LTCa ML) e largura do terço caudal do menisco medial (LTCa MM).

A área total dos meniscos laterais e mediais não apresentou diferença estatística significante (Fig. 5), entretanto a área do terço cranial mostrou diferença estatística, tendo o menisco medial apresentado média de $3,140 \mathrm{~mm}^{2}$, enquanto o menisco medial mostrou média de $2,463 \mathrm{~mm}^{2}$ (Fig. 6).

Os valores obtidos na mensuração de comprimento, espessura e largura dos ligamentos

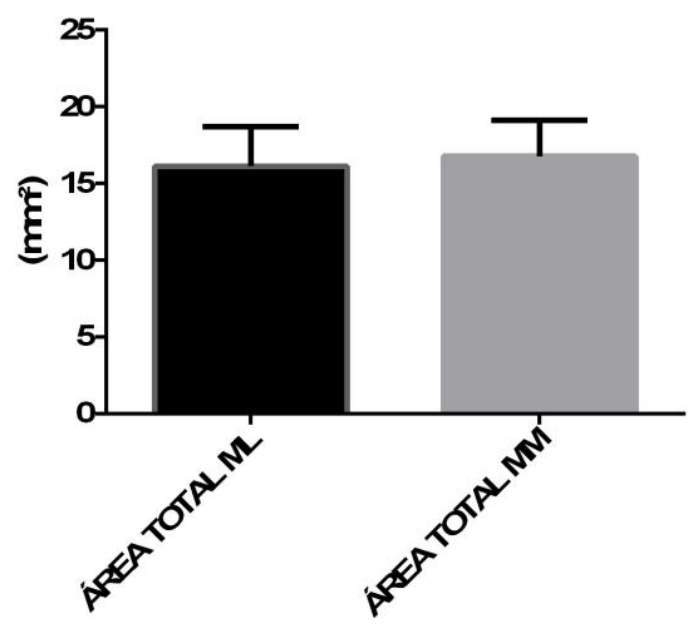

meniscais foram igualmente analisados, e calculados seus valores médios e respectivos desvio-padrão (Tab. 2).

Analisando-se os valores obtidos na mensuração do ligamento transverso dos meniscos medial e lateral, foi demonstrada diferença estatística apenas para largura e espessura (Fig. 7).

Figura 5. Médias e desvio-padrão das áreas totais do menisco lateral (ML) e do menisco medial (MM) de equinos. 


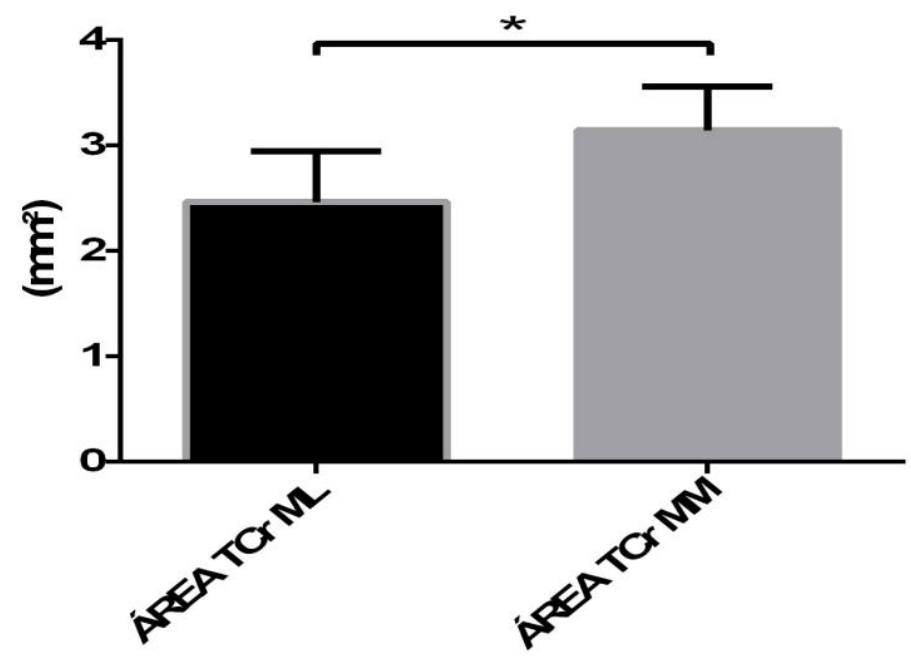

Figura 6. Valores médios e desvio-padrão das áreas do terço cranial (TCr) dos meniscos lateral (ML) e medial (MM) de equinos.

Tabela 2. Valores médios obtidos na mensuração dos ligamentos meniscais e seus respectivos desviospadrão (C - comprimento; L - largura; E - espessura) *Apresentaram diferença estatisticamente significante

\begin{tabular}{cccc}
\hline & & Menisco medial & Menisco lateral \\
\hline \multirow{2}{*}{ Ligamento transverso } & $\mathrm{C}$ & $20,850 \pm 3,964$ & $24,425 \pm 5,347$ \\
& $\mathrm{~L}^{*}$ & $10,205 \pm 1,809$ & $12,169 \pm 1,481$ \\
& $\mathrm{E}^{*}$ & $2,772 \pm 0,971$ & $4,122 \pm 1,743$ \\
Ligamento do corno cranial & $\mathrm{C}$ & $18,67 \pm 5,0166$ & $18,104 \pm 3,0203$ \\
& $\mathrm{~L}$ & $10,414 \pm 3,134$ & $10,330 \pm 2,522$ \\
& $\mathrm{E}$ & $2,084 \pm 0,667$ & $1,893 \pm 0,529$ \\
\hline
\end{tabular}

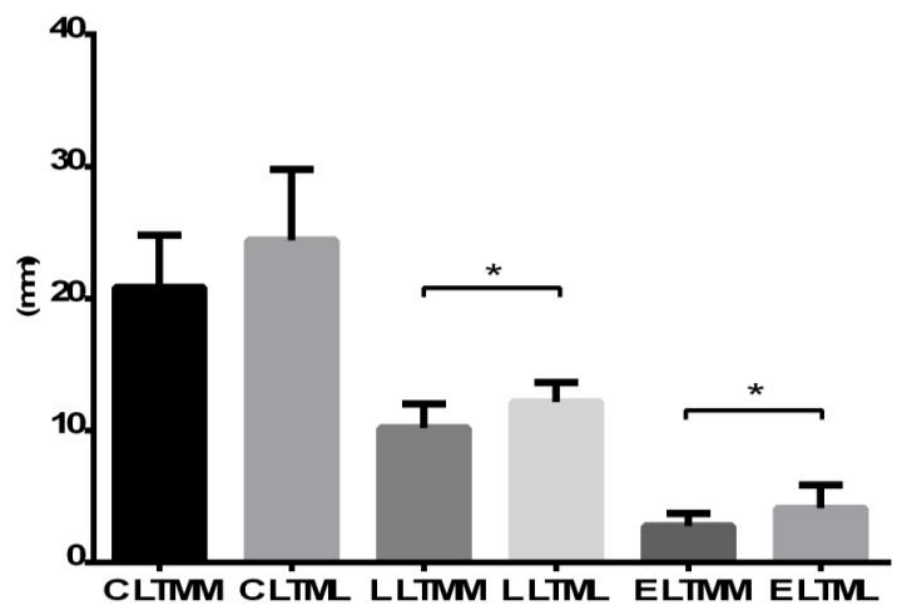

Figura 7. Valores médios e desvio-padrão das medidas de meniscos lateral e medial de equino, em que foram avaliados: comprimento do ligamento transverso do menisco medial (CLTMM), comprimento do ligamento transverso do menisco lateral (CLTML), largura do ligamento transverso do menisco medial (LLTMM), largura do ligamento transverso do menisco lateral (LLTML), espessura do ligamento transverso do menisco medial (ELTMM) e espessura do ligamento transverso do menisco lateral (ELTML). O ligamento do corno cranial não apresentou diferença estatística em todas as medições realizadas. 


\section{Morfometria dos meniscos...}

\section{DISCUSSÃO}

A articulação femorotibial é classificada morfológica e funcionalmente como condilar e biaxial, respectivamente. Desempenha movimentos de flexão, extensão e de rotação. A rotação ocorre medialmente, exercendo maior pressão dos côndilos do fêmur sobre os meniscos mediais. Os resultados obtidos permitiram a identificação, por meio da morfometria, de valores mais uniformes para o menisco lateral, o que possibilita inferir que este possa estar menos vulnerável a determinadas situações de estresse do que o menisco contralateral. O menisco medial possui maior circunferência externa em relação ao menisco lateral e, consequentemente, maior área de contato com os côndilos femorais e tibiais. Essa maior cobertura da superfície articular medial da tíbia pode receber maior pressão do côndilo do fêmur em conformidade com os relatos de maior incidência de lesões sobre o menisco medial.

Em um estudo, notou-se que, em 110 casos de lesões de menisco diagnosticadas, $79 \%$ envolviam o corno cranial do menisco medial, ao contrário do cão ou do homem, nos quais essas lesões são geralmente associadas aos ligamentos cruzados e/ou colaterais (Almeida et al., 2004; Walmsley, 2005; Murlimanju et al., 2010). Walmsley (2005) sugere que a compressão do corno cranial do menisco medial durante a hiperextensão da articulação é o principal mecanismo da lesão meniscal.

Os dados obtidos neste experimento podem sustentar os achados de Almeida et al. (2005), Walmsley (2005), Murlimanju et al. (2010), em que o menisco medial é o mais envolvido nos casos de lesões articulares em cavalos. O menisco medial possui maior circunferência externa em relação ao menisco lateral, sendo a possível causa de compressão do corno cranial do menisco medial durante a hiperextensão da articulação, que é o principal mecanismo da lesão meniscal observada em um estudo realizado em 2005 por Walmsley. Uma análise do deslocamento meniscal por meio da hiperextensão do joelho mostrou que nos $10^{\circ}$ finais (entre $150^{\circ}$ e $160^{\circ}$ ), o corno cranial do menisco medial apresenta maior mobilidade quando comparado ao corno cranial do menisco lateral. Assim, é possível que o corno cranial do menisco medial possa ficar preso entre os côndilos femoral e tibial, favorecendo, desse modo, a lesão nesse local. (Fowlie et al., 2011).

Não foram encontradas diferenças entre os ligamentos dos cornos craniais entre os meniscos lateral e medial, entretanto o ligamento transverso apresentou diferença significante no local da inserção, o que poderia contribuir para a mobilidade desigual entre os meniscos.

Danos no menisco medial têm sido associados também a lesões císticas subcondrais no côndilo femoral medial (Hendrix et al., 2010), porém não há ainda evidências de que tais alterações possam ser uma causa ou uma consequência sobre o menisco afetado. As espessuras dos terços médio e caudal maiores para o menisco lateral podem ratificar que o menisco medial, por ter maior circunferência de contato e menores espessuras, em 2/3 de sua área, estaria mais predisposto a sofrer danos quando submetido a situações de estresse, conforme relatado por Fowlie e colaboradores (2011). Novos estudos das propriedades biomecânicas, incluindo ensaios de tração, de resistência, de coeficientes de rigidez e de elasticidade, poderão ser úteis para determinar possíveis diferenças entre os meniscos lateral e medial em equinos.

\section{CONCLUSÕES}

Os resultados deste estudo mostraram uma relação importante nos dados morfométricos, com o menisco medial apresentando maior área do terço cranial e maior circunferência externa, seguido de menores espessura e largura dos terços médio e caudal em relação ao menisco lateral.

\section{REFERÊNCIAS}

ALMEIDA, S.K.S.; DEMORAES, A.S.R.; TASHIRO, T. et al. Morphometric study of menisci of the knee joint. Int. J. Morphol., v.22, p.181-184, 2004.

ARNOCZKY, S.P.; WARREN, R.F. The microvasculature of the meniscus and its response to injury. Am. J. Sports Med., v.11, p.131-141, 1983. 
FOWLIE, J.G.; ARNOCZKY, S.P.; LAVAGNINO, M.; STICK, J.A. Stifle extension results in differential tensile forces developing between abaxial and axial components of the cranial meniscotibial ligament of the equine medial meniscus: a mechanistic explanation for meniscal tear patterns. Equine Vet. J., v.44, p.554-558, 2012.

FOWLIE, J.G.; ARNOCZKY, S.P.; STICK, J.A.; PEASE, A.P. Meniscal translocation and deformation throughout the range of mo

tion of the equine Stifle joint: an in vitro cadaveric study. Equine Vet. J., v.43, p.259-264, 2011.

GUPTE, C.M.; BULL, A.M.; MURRAY, R.; AMIS, A.A. Comparative anatomy of the meniscofemoral ligament in humans and some domestic mammals. Anat. Histol. Embryol., v.36, p.47-52, 2007.

HENDRIX, S.M.; BAXTER, G.M.; McLLWRAITH, C.W. et al. Concurrent or sequential development of medial meniscal and subchondral cystic lesions within the medial femorotibial joint in horses (1996-2006). Equine Vet. J., v.42, p.5-9, 2010.
MURLIMANJU, B.V.; NAIR N.; PAI, S.R. et al. Morphometric analysis of the menisci of the knee joint in south indian human fetuses. Int. J. Morphol., v.28, p.1167-1171, 2010.

OLIVEIRA, H.C.S.; GALI, J.C.; CAETANO, E.B. Relações anatômicas entre as inserções femorais dos ligamentos meniscofemoral de Wrisberg e cruzado posterior. Rev. Bras. Ortop., v.48, p.412-416, 2013.

WALMSLEY, J.P. Diagnosis and treatment of ligamentous and meniscal injuries in the equine stifle. Vet. Clin. N. Am. Equine Pract., v.21, p.651-672, 2005.

WALMSLEY, J.P. Vertical tears of the cranial horn of the meniscus and its cranial ligament in the equine femorotibial joint: 7 cases and their treatment by arthroscopic surgery. Equine Vet. J., v.27, p.20-25, 1995. 